\title{
Metabolomics reveals changes in metabolite concentrations and correlations during sexual development of Eurotium cristatum (synonym: Aspergillus cristatus)
}

\author{
Ren CG ${ }^{1,2,3}$, Tan $\mathrm{YM}^{3,4}$, Ren $\mathrm{XX}^{6}$, Liu $\mathrm{YX}^{3,4}$ and Liu $\mathrm{ZY}^{5 *}$ \\ ${ }^{1}$ College of Agriculture, Guizhou University, Guiyang, Guizhou 550025, China \\ ${ }^{2}$ Guizhou Institute of Biology, Guiyang, Guizhou 550009, China \\ ${ }^{3}$ Guizhou Key Laboratory of Agricultural Biotechnology, Guiyang, Guizhou 550006, China \\ ${ }^{4}$ Guizhou Institute of Biotechnology, Guiyang, Guizhou 550006, China \\ ${ }^{5}$ Guizhou Academy of Agricultural Sciences, Guiyang, Guizhou 550006, China \\ ${ }^{6}$ Ecological Engineering College, Guizhou University of Engineering Science Bijie, Guizhou 551700, China
}

Ren CG, Tan YM, Ren XX, Liu YX, Liu ZY 2017 - Metabolomics reveals changes in metabolite concentrations and correlations during sexual development of Eurotium cristatum (synonym: Aspergillus cristatus). Mycosphere 8(10), 1626-1639, Doi 10.5943/mycosphere/8/10/3

\begin{abstract}
Eurotium cristatum is the main probiotic fungus involved in fermentation of Chinese Fuzhuan brick tea, and the amount of E. cristatum is an important indicator of tea quality. Sexual development of $E$. cristatum consists of three main stages, hypha and ascogonium (at 36 hours post-inoculation), cleistothecium formation (at 48 hours), and cleistothecium ripening (at 72 hours), and each stage exhibits a unique metabolite profile. In this study, developmental changes were analyzed by GC-MS based untargeted metabolomics. Ninety-nine metabolites involved in multiple biochemical pathways were identified. Principal component analysis separated developing $E$. cristatum into 36, 48 and 72 hour groups. Hierarchical cluster analyses showed that the relative contents of organic acids and amino acids peaked at 36 hours and decreased thereafter, while alcohol and carbohydrate levels peaked at 48-72 hours. Statistical analysis revealed 401 significant metabolite-metabolite correlations (Pearson's $|r|>0.8$ at a false discovery rate $\leq 0.05$ ), of which 213 were positive and 188 negative. The early increases in organic acid and amino acid metabolites as well as certain phosphate and amine metabolites favor ascogonium formation. Conversely, increased alcohol and carbohydrate concentrations favor cleistothecium formation. These results provide a foundation for further exploration of the biochemical mechanism underlying sexual development in E. cristatum.
\end{abstract}

Key words - Eurotium cristatum - Fungus - Metabolite - metabolite correlation - Metabolomic

\section{Introduction}

Eurotium cristatum (synonym: Aspergillus cristatus), termed the "Golden Flower Fungus," is the main probiotic fungus involved in the fermentation of Chinese Fuzhuan tea, a popular dark tea that has been produced for more than 100 years (Liu et al. 1991, Ge et al. 2016). Fuzhuan brick tea is rich in trace elements and amino acids, and has demonstrated lipid-lowering, weight loss, and antioxidant activities. It is considered "The tea of life" by herdsmen in regions such as Xinjiang, Gansu, and Ningxia. The unique taste and health benefits are due to microbial metabolism during 
fermentation (Mo et al. 2005, Mo et al. 2008, Liu et al. 1991). Eurotium cristatum can produce sexual spores under low osmotic pressure and asexual spores under high osmotic pressure. Thus, $E$. cristatum is both a commercially important fungal species and a suitable model for evaluating the mechanisms of spore production (Liu et al. 1991, Ge et al. 2016).

Many early studies revealed that fungus morphogenesis is related to the profile of six broad classes of metabolites: amino acids, carbohydrates, organic salts, alcohols, organic acids and oxylipin metabolites. Increase in glucose, lactose, glycine and glycerol favor cleistothecium formation. Mannitol is essential for the development of Aspergillus fischeri, while oxylipins can regulate the timing and balance between sexual and asexual spore development. Some alcohols (such as tyrosol, dodecanol, farnesol and glycerol), specific carbon sources (such as D-glucose, glucose and sucroseserum) and $\mathrm{N}$-acetylglucosamine play important roles in the morphological transition of Candida albicans (Han et al. 2003, Nickerson et al. 2006, Han et al. 2012, Wyatt et al. 2014), another important industrial microorganism used in Fuzhuan tea production. To explore how dynamic changes in metabolites regulate the sexual development of E. cristatum, we measured a broad array of metabolite concentrations during sporulation. Mycelia or spore samples from $E$. cristatum were collected at 36, 48 and $72 \mathrm{~h}$. post inoculation and GC-MS based metabolomics was used to investigate changes in metabolic composition during sexual development. Correlations among individual metabolites were in investigated to reveal the regulatory mechanisms governing these changes in metabolite profile. These results may help in the development of methods to control E. cristatum spore production for improving the quality and economic value of Fuzhuan tea.

\section{Materials \& Methods}

\section{Strains, media and culture conditions}

A laboratory strain of E. cristatum (No: GZAAS 20.1005) was isolated from Fuzhuan brick tea and propagated from a single spore at the Guzhou Key Laboratory of Agricultural Biotechnology. MYA medium with $5 \% \mathrm{NaCl}$ (20 g malt extract, $20 \mathrm{~g}$ yeast extract powder, $30 \mathrm{~g}$ sucrose, $18 \mathrm{~g}$ agar powder, $50 \mathrm{~g} \mathrm{NaCl}$ in $1000 \mathrm{~mL}$ water) was used for culture of hyphae and cleistothecia at $28{ }^{\circ} \mathrm{C}$ for 3 days. MYA medium with $17 \% \mathrm{NaCl}(\mathrm{NaCl} 170 \mathrm{~g} / \mathrm{L})$ was used for pure culture of conidia at $28^{\circ} \mathrm{C}$ for 5 days.

\section{Sample preparation}

The conidia were eluted in sterile deionized water and filtered through sterile absorbent cotton to remove mycelia. The final conidia suspension at $10^{6} / \mathrm{mL}$ was stored at $4{ }^{\circ} \mathrm{C}$. Subsequently, a $200 \mu \mathrm{L}$ sample was cultured on a cellulose membrane in $5 \% \mathrm{NaCl}$ MYA media at $28{ }^{\circ} \mathrm{C}$ for $36-72 \mathrm{~h}$. Samples from the three developmental stages were collected separately at 36,48 and $72 \mathrm{~h}$. Eighteen collected samples (six biological replicates at each point in time) were frozen in liquid nitrogen for $10 \mathrm{~min}$ and stored in an ultra-low temperature freezer $\left(-80{ }^{\circ} \mathrm{C}\right)$ until further processing. The morphology of E. cristatum at the three development stages (36, 48 and $72 \mathrm{~h}$.) was assessed by scanning electron microscopy.

\section{Main chemicals and instruments}

Methanol and methoxy solutions were purchased from Merck (Damstadt, Germany). The internal standards for nonadecylic acid, aminopropionic acid and N,O-Bis (trimethylsilyl) trifluoroacetamide were purchased from Fluka (Steinheim, Germany). The standard samples for C8-C20 and C21-C40 were purchased from Fluka (Chemika, Switzerland). All other reagents were of analytical grade. The GC-MS system consisted of an Agilent GC7890 gas chromatograph coupled to a MSD5975 mass selective detector electrospray ionization spectrometer operating at 70 eV (Agilent Technologies, USA).

\section{Metabolite extraction and derivatization}


The frozen samples were ground to obtain a fine powder, and 100-mg was used for each metabolite extraction. Extraction and derivatization methods were described previously (Ding et al. 2009, Ding et al. 2010).

\section{Data processing and statistical analysis}

The samples were processed using a global accepted mass spectrometry-based platform with GC-MS. Data processing methods were described previously (Ding et al. 2009, Ding et al. 2010). The original data were transformed into CDF format (NetCDF) using Agilent GC/MS 5975 Data Analysis software and processed using XCMS software (www.bioconductor.org). The results from XCMS were exported to EXCEL for further analysis. The metabolites were identified using the automated mass spectral DE convolution and identification system (AMIDS), while mass spectra were identified using National Institute of Standards and Technology (NIST) and Wiley libraries. After all data were normalized, principal component analysis (PCA) was conducted using SIMCAP software (Mohler et al. 2008, Smith et al. 2006, Rao et al. 2016). The metabolites were mapped to metabolic pathways using the KEGG annotation information system (http://www.genome.jp/kegg/pathway.html). Heat maps were constructed using Euclidian distances and complete linkage grouping with the $\mathrm{R}$ language package of Pheatmap (www.r-project.org). Metabolic correlation maps were constructed using Cytoscape 3.2.0 software (http://www.cytoscape.org/)

\section{Results}

\section{Morphological changes during sexual development of $E$. cristatum}

E. cristatum (GZAAS 20.1005) was isolated from Fuzhuan brick tea and cultured on 5\% MYA medium at $28{ }^{\circ} \mathrm{C}$ for $36-72 \mathrm{~h}$. Colonies were white at $36 \mathrm{~h}$. post-inoculation, yellow with white edges at $48 \mathrm{~h}$. and golden yellow at $72 \mathrm{~h}$. Scanning electron microscopy showed that $E$. cristatum produced hyphae and ascogonia at $36 \mathrm{~h}$. Over the next $12 \mathrm{~h}$., cleistothecia began to form, reaching $40 \mathrm{~mm}$. in diameter by $48 \mathrm{~h}$. and $90 \mathrm{~mm}$ at $72 \mathrm{~h}$. (Fig. 1).
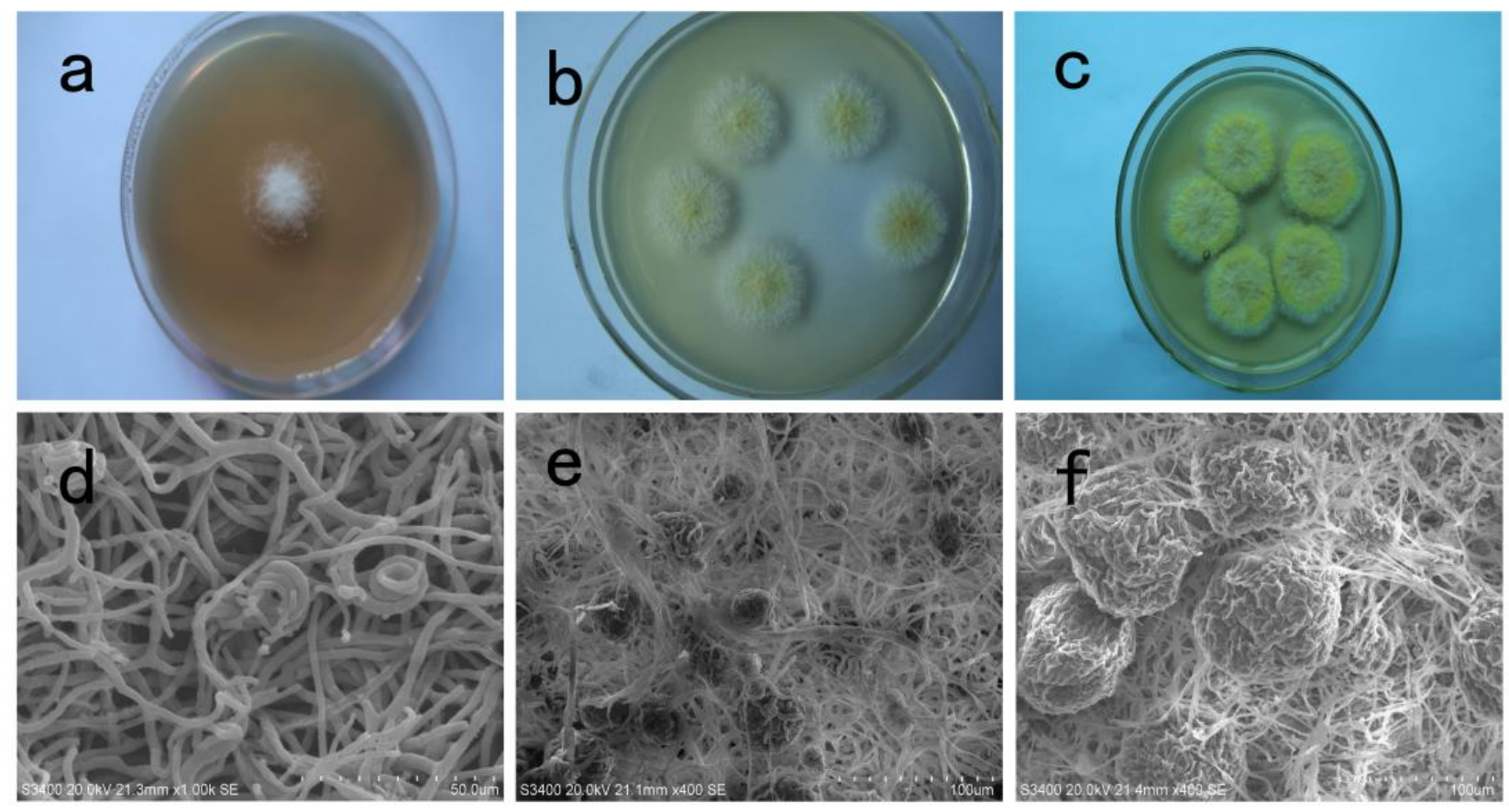

Figure 1 - Sexual development of E. cristatum. (a-c) Colony morphology of E. cristatum at 36, 48, and $72 \mathrm{~h}$. after plating. (d) Hyphae and ascogonia of E. cristatum at $36 \mathrm{~h}$. (e-f) Cleistothecia at 48 and $72 \mathrm{~h}$. 


\section{Changes in metabolite profile during sexual development of $E$. cristatum}

Based on morphological observations suggesting three developmental stages (Fig. 1), colonies were sampled at 36, 48 and $72 \mathrm{~h}$. and metabolite profiles were investigated using an untargeted global metabolomics platform with GC-MS analysis. From the total ion current chromatograms (Fig. 1), a total of 99 metabolites were confirmed by National Institute of NIST and Wiley Registry mass spectral libraries. These 99 metabolites were classified into eight major groups covering multiple metabolic pathways. The first group contained 34 organic acid metabolites, the second 24 amino acids, the third 13 carbohydrate metabolites, the forth 9 alcohols. The fifth to seventh groups contained 6 fatty acid, amine, and phosphate metabolites, respectively. One phenol metabolite was also identified (Table 1). PCA of the entire group of 99 metabolites yielded two principal components explaining $48.3 \%$ of the overall variance in metabolite profile during sexual development, $30.1 \%$ for PC1 and $18.2 \%$ for PC2 (Fig. 2). Thus, PCA scores revealed that the samples could be clearly separated during sexual development (Fig. 2a). The KEGG showed that the 99 identified metabolites covered 133 pathways, including "Biosynthesis of plant secondary metabolites", "Aminoacyl-tRNA biosynthesis", "Mineral absorption", "ABC transporters", "Protein digestion and absorption", "Microbial metabolism in diverse environments" and "Biosynthesis of secondary metabolites" (Table 1).


Figure 2 - PCA plots and loading plots of the metabolite profiles for each stage of E. cristatum sexual development. (a) In the scores plot, the $95 \%$ confidence interval (Hotelling's T2 ellipse) was SIMCA-P $+13.0, \mathrm{R} 2 \mathrm{X}[1]=0.301$, and R2X [2] $=0.182$. Observations outside the confidence ellipse are considered outliers. The scores plot indicates that samples can be clearly separated in three distinct cultivation period groups $(36,48$, and $72 \mathrm{~h}$ ). (b) PCA loading plots generated from all 99 metabolites identified from different samples.

\section{One way analysis of variance (ANOVA) and hierarchical cluster analyses of metabolites during sexual development of $E$. cristatum}

The levels of individual intracellular metabolites at $36 \mathrm{~h}$. were compared to levels at 48 and $72 \mathrm{~h}$. by one way ANOVA with adjustment for multiple hypothesis testing. Results revealed that 43 metabolites were differentially expressed at $48 \mathrm{~h}$. and 51 metabolites were differentially expressed at $72 \mathrm{~h}$. (Table 2). The 43 metabolites differentially expressed in the $48 \mathrm{~h}$. group compared to the 36 h. group included 15 amino acids and amino acid derivatives, 14 organic acids, 6 carbohydrates, 5 alcohol metabolites, and 3 phosphates, while the 51 differentially expressed at $72 \mathrm{~h}$. compared to 36 h. included 24 organic acids, 9 amino acids, 7 carbohydrates, 4 alcohol metabolites, 5 phosphates, 1 amine and 1 fatty acid. The majority of these differentially expressed metabolites are involved in amino acid and organic acid metabolism Hierarchical cluster analysis (HCA) showed that the majority of organic acids and amino acids were up regulated at $36 \mathrm{~h}$. and down regulated thereafter 
with no difference between 48 h. and 72 h. groups. In contrast, the alcohol metabolites were gradually up regulated during sexual development from $36 \mathrm{~h}$ to 48 and $72 \mathrm{~h}$. Similarly, carbohydrate metabolites were up regulated at $48 \mathrm{~h}$. compared to $36 \mathrm{~h}$. Some carbohydrates were down regulated at $72 \mathrm{~h}$. compared to $48 \mathrm{~h}$., while others were up regulated at $72 \mathrm{~h}$. compared to 48 h. (Fig. 3).

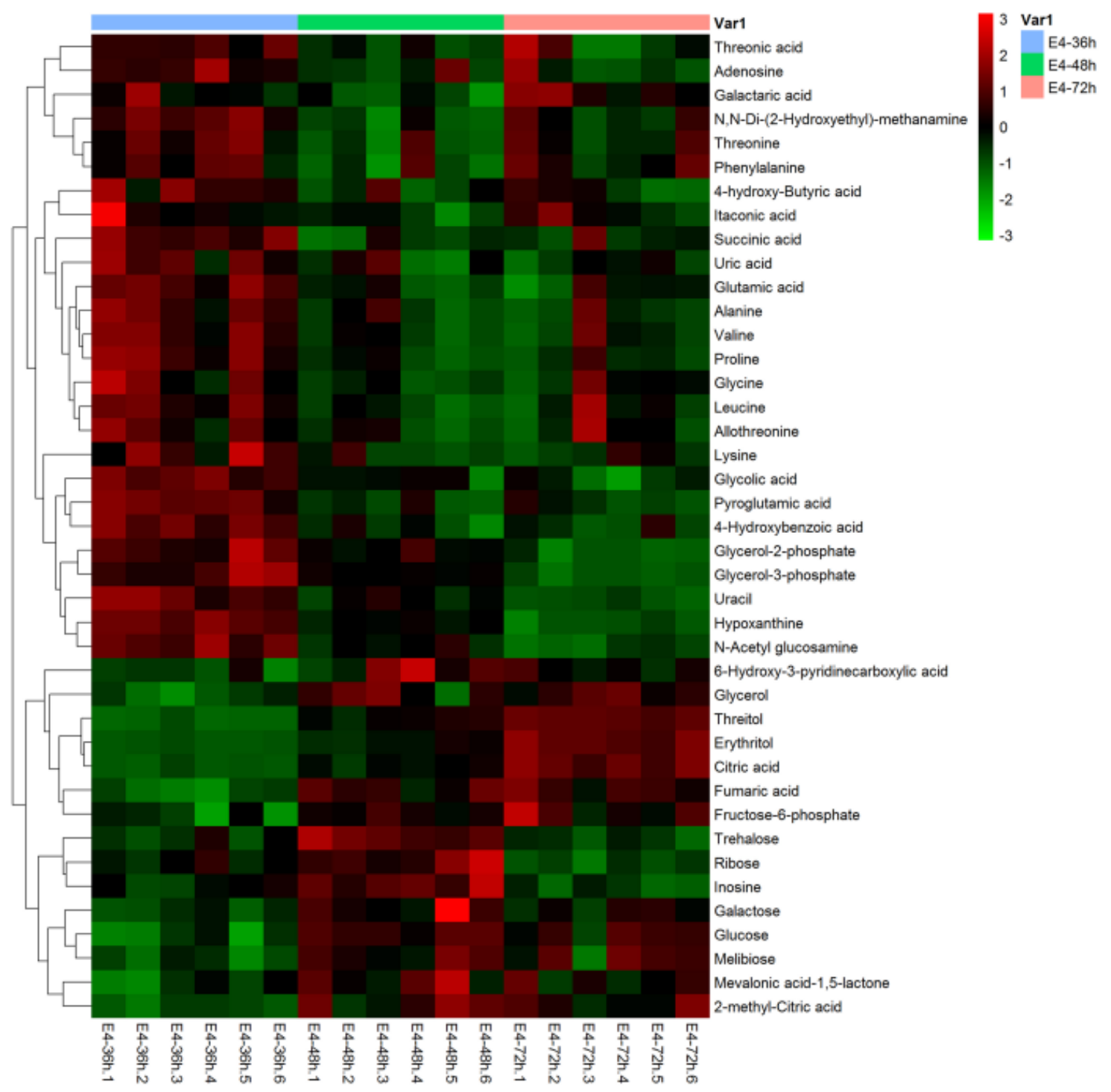

Figure 3 - Hierarchical cluster analysis (HCA) of E. cristatum (E4) metabolites differentially expressed among 36, 48, and $72 \mathrm{~h}$. cultivation periods. Red squares in the heat map indicate increases in intracellular metabolite concentration, while green squares indicate decreases in intracellular metabolite concentration.

\section{Correlation analysis during sexual development of $E$. cristatum}

Pearson correlation analysis revealed 1252 individual metabolite-metabolite correlations during sexual development of $E$. cristatum, of which 710 were positive and 542 were negative (Fig. 4a). Of these correlations, 401 were significant at $\mathrm{P}<0.05,213$ positive and 188 negative (Fig. 4b). Amino acids were most numerous in the significant positive correlation group, followed by organic acids, phosphates, and amine metabolites, while carbohydrates predominated in the significant negative correlation group, followed by organic acids, alcohols, and phosphate metabolites. These results suggest that amino acids, carbohydrates, organic acids, alcohols, amines and phosphate metabolites have unique functions in the different phases of E. cristatum sexual development. 

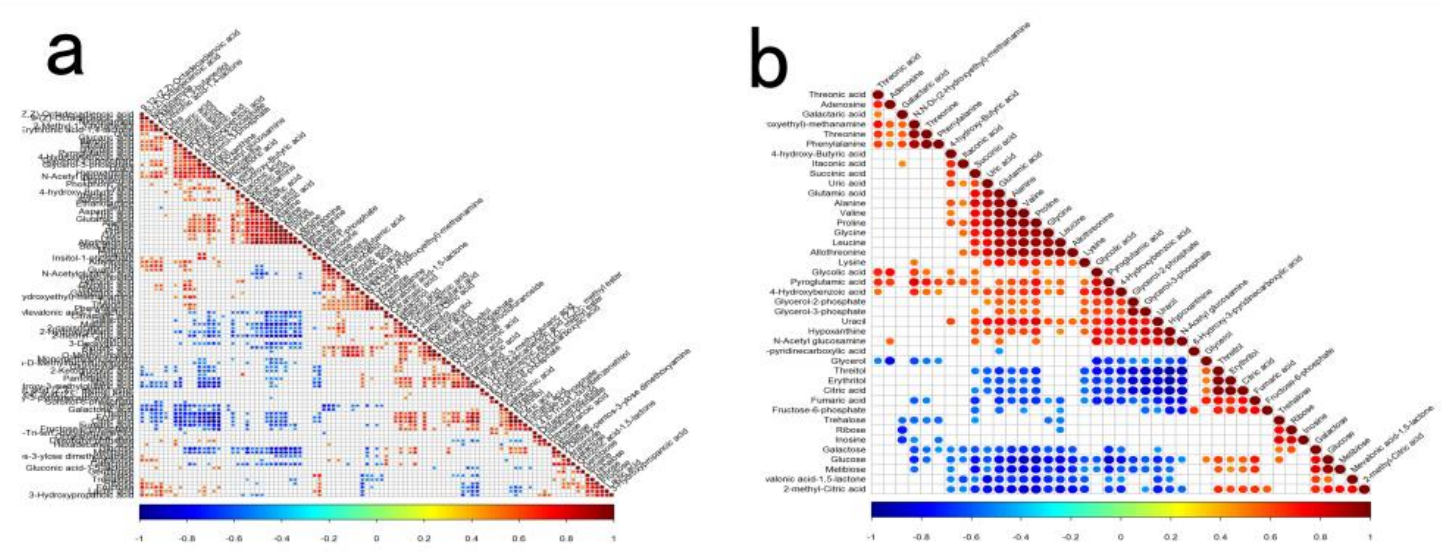

Figure 4 - Metabolite-metabolite correlation analysis. Positive correlations are shown in red and negative correlations in blue. (a) All metabolite-metabolite correlations are expressed as Pearson's correlation coefficients (r). (b) Significant metabolite-metabolite correlations ( $\mathrm{r}$ values with $\mathrm{P}<$ $0.05)$.

Table 1 Intracellular metabolites of E. cristatum (synonym: Aspergillus cristatus) identified by GC-MS

\begin{tabular}{|l|l|l|}
\hline Classification & No & Intracellular metabolites \\
\hline Organic acids & 34 & $\begin{array}{l}\text { Pyruvic acid, Lactic acid, Glycolic acid, 3-Hydroxypropanoic acid, } \\
\text { Malonic acid, 2-oxoisocaproic acid, 4-hydroxy-Butyric acid, Benzoic } \\
\text { acid, Phosphoric acid, Succinic acid, Glyceric acid, Itaconic acid, Glutaric } \\
\text { acid, Malic acid, Threonic acid, Citramalic acid, 6-Hydroxy-3- } \\
\text { pyridinecarboxylic acid, Tetronic acid, 2-Hydroxyglutaric acid, 3- } \\
\text { Hydroxy-3-methylglutaric acid, 4-Hydroxybenzoic acid, Aconitic acid, } \\
\text { 2-Ketogluconic acid, Ribonic acid, Citric acid, Fumaric acid, 2-methyl- } \\
\text { Citric acid, Diisobutyl-phthalate, Galactonic acid, Pantothenic acid, } \\
\text { Galactaric acid, Glucaric acid, Uric acid, Mevalonic acid-1, 5-lactone }\end{array}$ \\
\hline $\begin{array}{l}\text { Amino acids } \\
\text { and derivatives }\end{array}$ & 24 & $\begin{array}{l}\text { Alanine, Valine, Leucine, Threonine, Proline, Glycine, Homoserine, } \\
\text { Serine, Allothreonine, Beta-Alanine, Pyroglutamic acid, Aspartic acid, } \\
\text { N-Acetylglutamic acid, Phenylalanine, Glutamic acid, Lysine, Tyrosine, } \\
\text { Uridine, Uracil, Inosine, Adenosine, Guanosine, Hypoxanthine, Guanine }\end{array}$ \\
\hline Carbohydrates & 13 & $\begin{array}{l}\text { Glucopyranose, Arabinose, Melibiose, Galactinol, Ribose, Fructose, } \\
\text { Glucose, Galactose, Trehalose, Gentibiose, alpha-D- } \\
\text { Methylfructofuranoside, Gluconic acid-1,5-lactone, Erythronic acid-1,4- } \\
\text { lactone }\end{array}$ \\
\hline Alcohols & 9 & $\begin{array}{l}\text { Glycerol, 2-Methyl-1, 3-butanediol, Erythritol, Threitol, Arabinitol, 3- } \\
\text { Deoxyglucitol, O-Methyl-inositol, Mannitol, myo-inositol }\end{array}$ \\
\hline Fatty acids & 6 & $\begin{array}{l}\text { Hexadecanoic acid, 9-Octadecenoic acid (Z) - methyl ester, 9,12- } \\
\text { Octadecadienoic acid (Z,Z) - methyl ester, 9,12- (Z,Z)-Octadecadienoic } \\
\text { acid, 9- (Z) -Octadecenoic acid, Octadecanoic acid }\end{array}$ \\
\hline Phenols & 1 & $\begin{array}{l}\text { Urea, Ethanolamine, N,N-Di-(2-Hydroxyethyl)-methanamine, } \\
\text { Nicotinamide, 2-Desoxy-pentos-3-ylose dimethoxyamine, N-Acetyl } \\
\text { glucosamine }\end{array}$ \\
\hline $\begin{array}{l}\text { Sorbitol-6-phosphate, Insitol-1-phosphate, Fructose-6-phosphate, } \\
\text { Monomethylphosphate, Glycerol-3-phosphate, Glycerol-2-phosphate }\end{array}$ \\
2,4,6-Tri-tert-butylbenzenethiol \\
\hline
\end{tabular}




\section{Discussion}

Filamentous fungi have a complex life cycle that includes spore germination, hypha formation, aerial hyphae production and finally emergence of sexual and asexual spores (Dyer \& O'Gorman 2012, Krijgsheld et al. 2013). Changes in morphology and metabolite profile during sexual development of E. cristatum are temporally regulated. Filamentous fungi have attracted great attention as models for both basic and applied research because of their complex morphological structures, development changes, numerous metabolic products, and metabolic regulatory networks (Etxebeste et al. 2010, Yu 2010, Dyer \& O'Gorman 2012, Han et al. 2012, , Ana et al. 2016). The high sensitivity, accuracy, and throughput capacity of metabolomics technologies have allowed for the analysis of complex metabolic changes associated with morphogenesis (Bino et al. 2004, Han et al. 2012). Indeed, metabolomics analysis has revealed that morphological development is associated with dynamic changes in metabolite profiles and metabolite networks (Bino et al. 2004, Ding et al. 2009, Roze et al. 2010, Han et al. 2012).

In the present study, metamorphosis of E. cristatum during sexual development as observed by scanning electron microscopy (Fig. 1) was strongly associated with changes in metabolite profile (Table 2). Indeed, PCA scores indicated that the morphologically distinct phases of sexual development could also be distinguished by metabolite composition (Fig. 2). One- way ANOVA (Table 2), hierarchical cluster analyses (Fig. 3), and correlation analysis (Fig. 4) identified six major metabolite groups (amino acids, organic acids, alcohols, carbohydrates, phosphates and amines) exhibiting characteristic changes during sexual development of E. cristatum. Of these, organic acids and amino acids constituted the largest groups (36.59\% and $29.27 \%$ of the total) and both exhibited significant down regulation during the transition from the hypha and ascogonium stage (at $36 \mathrm{~h}$.) to the cleistothecium formation stage (at $48 \mathrm{~h}$.), suggesting substantial contributions in the early stage of sexual development. In contrast, the less numerous carbohydrates $(14.63 \%$ of the total) and alcohols (7.32\%) were up regulated during this transition (Fig. 3). Previous research has shown that morphogenesis of other filamentous fungi is related to the profile of amino acids, carbohydrates, organic salts, alcohols, organic acids and oxylipins among other metabolites. However, most previous studies focused on single metabolites, such as glucose, glycine, glycerol, mannitol, oxylipins, $\mathrm{N}$-acetylglucosamine, different carbon sources and proline (, Chen et al. 2004 , Nickerson et al. 2006, Han et al. 2011, Wyatt et al. 2014) but there have been no reports on the developmental changes in large metabolite categories and associations among categories during morphogenesis, The early metabolomics studies of Han et al. (year) revealed that 19 intracellular metabolites were upregulated during the morphological transition of Candida albicans, mainly amino acids (such as glycine, proline and phenylalanine), fatty acids (such as caprinate and myristate) and organic acid salts (such as malate and succinate), consistent with key roles in morphological transition (Han et al. 2012). There appear to be substantial differences in the regulation of these metabolite classes during morphogenesis among species such as E. cristatum and Aspergillus nidulas, which show distinct developmental regulation of glucose, galactose, glycerol, proline and $\mathrm{N}$-acetylglucosamine among other metabolites (Han et al. 2003). Other studies have identified metabolites related to sexual development of E. cristatum, such as organic acids (threonic acid, uric acid, glutamic acid, citric acid and 2-methyl citric acid), amino acids (lysine, hypoxanthine and valine), alcohols (threitol and erythritol), carbohydrates (melibiose, trehalose and ribose), organic salts (fructose-6-phosphate and glycerol-3-phosphate) and mevalonic acid-1,5lactone. However, specific functions in morphogenesis of E. cristatum are currently unclear. Here we provide preliminary evidence for reciprocal regulation between metabolite classes that appears critical for metamorphosis.

The spores of E. cristatum formed hyphae and slow growing ascogonia within $24 \mathrm{~h}$. of inoculation. During this period, fungi entered logarithmic growth, and mycelium curling resulted in the formation of ascogonia. In addition, organic acids and amino acids were rapidly synthesized (Fig. 3). However during the transition to the cleistothecia formation stage $(36-48 \mathrm{~h}$.), levels of organic and amino acids fell significantly. Similarly, during the morphological transition of $C$. albicans, the concentrations of amino acids and organic acids first increase in spores and then 
decrease in hyphae (Han et al. 2003). Cleistothecia development requires greater nutrition and energy. Thus, carbohydrate accumulation and metabolism accelerate during cleistothecia development to generate energy for complex biosynthetic processes (Han et al. 2012).

During sexual development, most saccharide metabolites and alcohols increased, while most phosphate metabolites gradually decreased (Fig. 3). High concentration of sugars can increase osmotic pressure, activating the high osmolarity glycerol (HOG) pathway, which in turn can induce accumulation of alcohols. Glycerol can help fungi quickly adapt to environmental changes, while some alcohols are required for normal sexual development (Blomberg \& Adler 1989, Chen et al. 2004, Chen \& Fink 2006, Nickerson et al. 2006, Wyatt et al. 2014,). Furthermore, glycerol $3-$ phosphate was shown to be a direct precursor of glycerol through dephosphorylation by glycerol3-phosphatase (G3 Pase) (Gancedo et al. 1968). We speculate that G3 Pase contributes to glycerol formation in E. cristatum as alcohols were upregulated concomitantly with downregulation of phosphates. Numerous reports on environmental factors affecting the development of fungi have documented changes in alcohols, carbohydrates, and amines during metamorphosis (Chen et al. 2004, Maidan et al. 2005b, Han et al. 2012). Thus metabolites such as organic acids, amino acids, sugars metabolites, alcohols and phosphates appear to be critical for morphogenesis of E. cristatum and other fungi under a variety of conditions.

Fatty acids are particularly versatile regulators of development, with functions in hypha growth, biofilm formation, morphological transformation, quorum sensing, and in determining the balance between asexual and sexual spore development (Kinderlerer 1993, Zhao \& Zheng 1995, Chen et al. 2004, Dimitrios \& Nancy 2007, Christensen \& Kolomiets 2011, Han et al. 2011, Han et al. 2012 ). We found that of all fatty acids examined, only 9-octadecenoic acid (Z)-methyl ester increased progressively with cultivation time, while the other 5 fatty acids showed no significant changes from 36 h. to 48 h. and were significantly downregulated at 72 h. (Fig. 5). Mazur et al. (1991) found that the fungal oxylipins $(\mathrm{psiB} \alpha)$ can stimulate sexual development in Aspergillus.
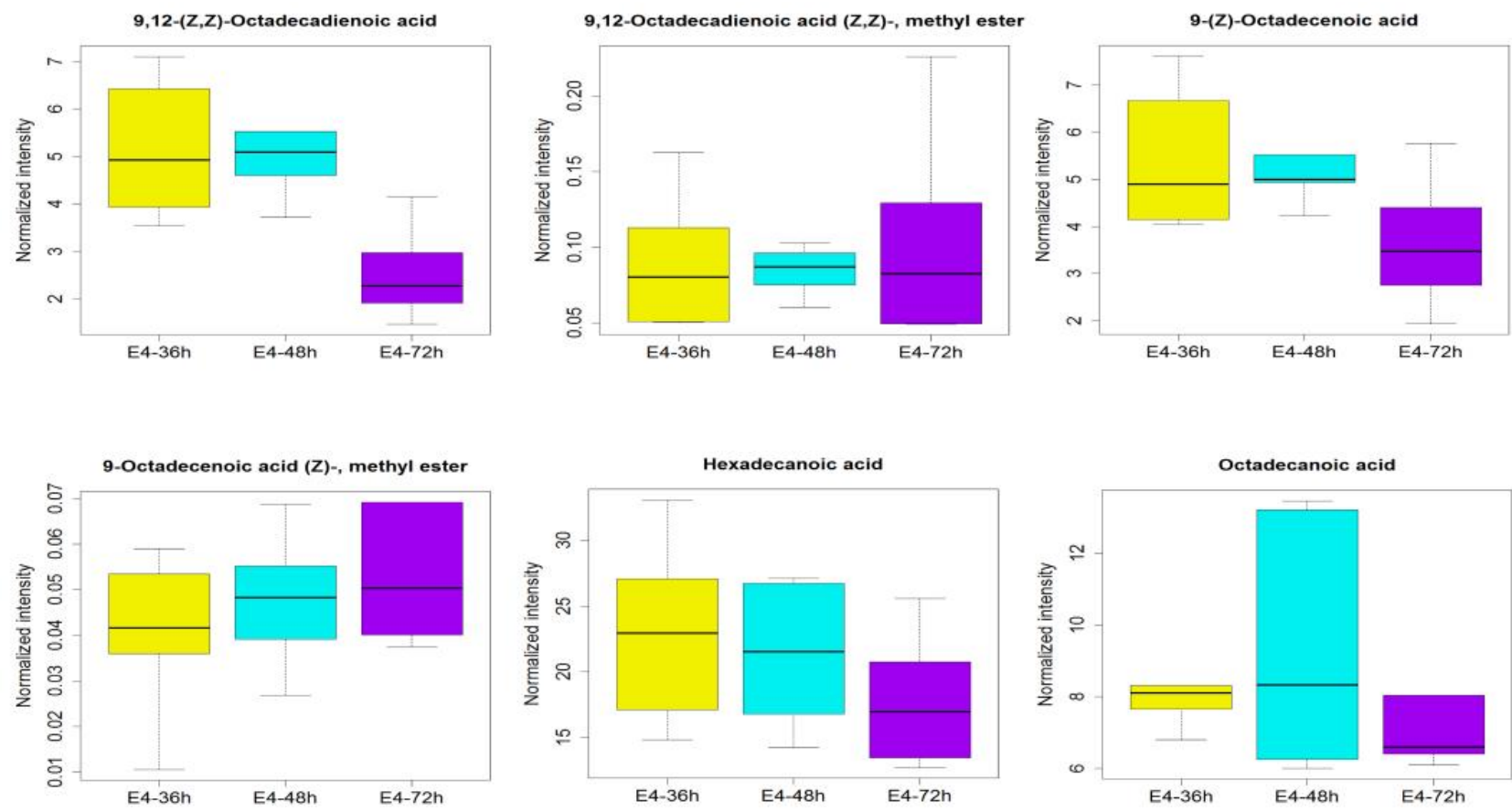

Figure 5 - Box plot visualization of changes in fatty acid metabolite levels in E. cristatum (E4) during sexual development (36, 48 and $72 \mathrm{~h}$.) 
Table 2 The intracellular metabolites identified of E. cristatum by GC-MS at different times

\begin{tabular}{|c|c|c|c|c|c|}
\hline Name & RI & $\mathrm{mz}$ & RT & $\begin{array}{l}\text { E4-48h vs E4-36h } \\
\text { pvalue }\end{array}$ & $\begin{array}{l}\text { E4-72h vs E4 36h } \\
\text { pvalue }\end{array}$ \\
\hline Threitol & 1530 & 217.1000061 & 759.329 & $1.16071 \mathrm{E}-05^{*}$ & $1.18584 \mathrm{E}-10^{*}$ \\
\hline Hypoxanthine & 1818.6 & 265.1000061 & 956.1205 & 7.96163E-06* & $1.2157 \mathrm{E}-07 *$ \\
\hline Trehalose & 2809.8 & 204.1000061 & 1460.215 & $0.00031471 *$ & 0.349443257 \\
\hline Pyroglutamic acid & 1533.6 & 156 & 763.822 & $0.000163375^{*}$ & $0.000230715^{*}$ \\
\hline Erythritol & 1519.2 & 217.1000061 & 752.906 & $0.000149647 *$ & 2.37915E-08 \\
\hline Fumaric acid & 1351.2 & 245 & 623.5765 & $0.000354645^{*}$ & $0.00014927^{*}$ \\
\hline Citric acid & 1844.1 & 273.1000061 & 970.5355 & $7.30441 \mathrm{E}-05^{*}$ & $1.07944 \mathrm{E}-07 *$ \\
\hline Glucose & 1934.2 & 319.1933977 & 1026.326 & $0.000213697 *$ & $0.005038044 *$ \\
\hline N-Acetyl glucosamine & 2116.8 & 205.1000061 & 1129.701 & $0.000840284 *$ & 7.37379E-06* \\
\hline 4-Hydroxybenzoic acid & 1633.3 & 267.0842842 & 834.121 & $0.000484827 *$ & $0.000333586^{*}$ \\
\hline Uracil & 1348.1 & 99 & 621.051 & $0.002246036^{*}$ & $9.86149 \mathrm{E}-06^{*}$ \\
\hline $\begin{array}{l}\mathrm{N}, \mathrm{N}-\mathrm{Di} \text {-(2-Hydroxyethyl)- } \\
\text { methanamine }\end{array}$ & 1430.6 & 160 & 686.158 & $0.000281421^{*}$ & $0.028782636^{*}$ \\
\hline Inosine & 2603 & 217.1000061 & 1370.676 & $0.001684504 *$ & 0.058634292 \\
\hline Proline & 1310.4 & 142.0753864 & 590.1685 & $0.001041392 *$ & $0.003753318 *$ \\
\hline Succinic acid & 1320.4 & 247.0992681 & 598.405 & $0.000553554^{*}$ & $0.008676202 *$ \\
\hline Glutamic acid & 1632.2 & 246.1000061 & 832.864 & $0.000634791 *$ & $0.00512178 *$ \\
\hline 2-methyl-Citric acid & 1862 & 287.0945742 & 982.4145 & $0.002521676^{*}$ & $0.002498419 *$ \\
\hline Glycolic acid & 1082.6 & 177.0026609 & 388.957 & $0.001593739 *$ & $0.000424529 *$ \\
\hline Glycerol-3-phosphate & 1784.7 & 299.1000061 & 934.438 & $0.009597488 *$ & 7.31762E-05* \\
\hline Valine & 1224.3 & 144.1000061 & 517.0325 & $0.001884233^{*}$ & $0.018174294 *$ \\
\hline Ribose & 1703 & 103 & 881.274 & $0.013464194 *$ & $0.008012298 *$ \\
\hline Leucine & 1281.6 & 158.1000061 & 565.855 & $0.00079941 *$ & 0.108244405 \\
\hline Glycerol & 1295.8 & 205 & 578.999 & $0.013520729 *$ & $0.00039579 *$ \\
\hline Melibiose & 2944 & 204.1000061 & 1515.389 & $0.001533409 *$ & $0.021086233 *$ \\
\hline Galactose & 1952.6 & 319.1909065 & 1036.921 & $0.015608135^{*}$ & $0.030939532 *$ \\
\hline Glycerol-2-phosphate & 1749 & 299.0853256 & 911.122 & $0.023353161 *$ & $9.52371 \mathrm{E}-05^{*}$ \\
\hline Alanine & 1109.7 & 116 & 414.018 & $0.009199788^{*}$ & $0.016215935^{*}$ \\
\hline Lysine & 1718 & 84.01578692 & 890.908 & $0.020785727^{*}$ & $0.025390484 *$ \\
\hline $\begin{array}{l}\text { Mevalonic acid-1,5- } \\
\text { lactone }\end{array}$ & 1376.4 & 145 & 644.4185 & $0.019265018^{*}$ & $0.046052188 *$ \\
\hline Threonine & 1307.5 & 117 & 587.979 & $0.016724637 *$ & 0.205469475 \\
\hline $\begin{array}{l}\text { 6-Hydroxy-3- } \\
\text { pyridinecarboxylic acid }\end{array}$ & 1577.8 & 268.0714223 & 795.435 & $0.031540129 *$ & $0.037306848 *$ \\
\hline Glycine & 1319 & 174.0989731 & 597.6325 & $0.017049102 *$ & 0.166689381 \\
\hline Phenylalanine & 1554.6 & 120 & 778.5835 & $0.025799365^{*}$ & 0.565063638 \\
\hline Fructose-6-phosphate & 2356.5 & 299.0882995 & 1249.244 & $0.008638952 *$ & $0.015174478 *$ \\
\hline 4-hydroxy-Butyric acid & 1241.1 & 116.9725448 & 531.563 & $0.028847427 *$ & $0.040789597 *$ \\
\hline Uric acid & 2128.4 & 441.2000122 & 1136.404 & 0.063330447 & $0.013860968 *$ \\
\hline Threonic acid & 1563.7 & 292.1000061 & 785.317 & $0.000960822 *$ & 0.215281455 \\
\hline Itaconic acid & 1359.1 & 259.023503 & 630.0095 & 0.05574631 & 0.497659779 \\
\hline Allothreonine & 1399.2 & 117 & 662.574 & $0.025451614^{*}$ & 0.238211839 \\
\hline Adenosine & 2621.3 & 245.086855 & 1378.7175 & $0.027060864 *$ & 0.064391629 \\
\hline
\end{tabular}


Table 2 Continued.

\begin{tabular}{|c|c|c|c|c|c|}
\hline Name & RI & $\mathrm{mz}$ & RT & $\begin{array}{l}\text { E4-48h vs E4-36h } \\
\text { pvalue }\end{array}$ & $\begin{array}{l}\text { E4-72h vs E4 36h } \\
\text { pvalue }\end{array}$ \\
\hline Galactaric acid & 2041.1 & 333.1000061 & 1087.941 & 0.053672117 & 0.346676714 \\
\hline Glutaric acid & 1408 & 186 & 610.2085 & 0.060245023 & $0.001769109^{*}$ \\
\hline $\begin{array}{l}\text { 2,4,6-Tri-tert.- } \\
\text { butylbenzenethiol }\end{array}$ & 1552.7 & 263.1857678 & 777.298 & 0.066186059 & 0.119872973 \\
\hline Citramalic acid & 1569.9 & 247.1000061 & 789.797 & 0.094487306 & 0.100758068 \\
\hline Fructose & 1906.5 & 103.0593474 & 1012.8055 & 0.112903575 & $0.008165376^{*}$ \\
\hline Homoserine & 1364.1 & 146 & 634.253 & 0.092456338 & 0.098653784 \\
\hline 3-Deoxyglucitol & 1813.2 & 103 & 952.845 & $0.007325045^{*}$ & $0.03784704 *$ \\
\hline Malonic acid & 1211.2 & 233 & 505.48 & 0.056921303 & $0.007323186^{*}$ \\
\hline 2-Methyl-1,3-butanediol & 1390.9 & 117 & 655.672 & 0.143148415 & $0.002504793 *$ \\
\hline Tetronic acid & 1580.1 & 292.1000061 & 797.1945 & $0.006665865^{*}$ & 0.675374655 \\
\hline Benzoic acid & 1251.7 & 178.9934686 & 540.413 & 0.17559599 & $0.001100196^{*}$ \\
\hline Tyrosine & 1897.1 & 179.1000061 & 1004.1995 & 0.125736641 & 0.61520412 \\
\hline Arabinose & 1687.7 & 103 & 871.0055 & 0.215121198 & 0.389734238 \\
\hline $\begin{array}{l}\text { alpha-D- } \\
\text { Methylfructofuranoside }\end{array}$ & 1801.5 & 217.1000061 & 944.501 & $0.041328516^{*}$ & 0.080911073 \\
\hline $\begin{array}{l}\text { 2-Desoxy-pentos-3-ylose } \\
\text { dimethoxyamine }\end{array}$ & 1777.3 & 231.1000061 & 929.408 & 0.194994803 & 0.617270109 \\
\hline Sorbitol-6-phosphate & 2403.1 & 299.0945637 & 1277.587 & 0.103582683 & 0.147176055 \\
\hline Glyceric acid & 1343.1 & 292.1000061 & 616.84 & 0.067880325 & 0.203913829 \\
\hline Serine & 1372.1 & 204.1000061 & 640.5865 & 0.077708032 & 0.68416448 \\
\hline 3-Hydroxypropanoic acid & 1150.8 & 176.9938643 & 451.248 & 0.152495913 & $0.028219969 *$ \\
\hline 2-Hydroxyglutaric acid & 1586 & 129 & 801.084 & 0.152395148 & $0.010144772 *$ \\
\hline Phosphoric acid & 1297.7 & 299.0980299 & 580.558 & 0.354383153 & 0.077810022 \\
\hline Aspartic acid & 1534.4 & 100 & 764.139 & 0.190885203 & 0.453868108 \\
\hline Urea & 1260.5 & 189.008732 & 552.317 & 0.408676417 & $0.019528125^{*}$ \\
\hline Malic acid & 1501.7 & 233.1000061 & 740.0755 & 0.127784651 & $4.02761 \mathrm{E}-06^{*}$ \\
\hline Galactonic acid & 1994 & 292 & 1060.689 & 0.386133621 & $0.003955594 *$ \\
\hline Glucaric acid & 2058.2 & 292.1000061 & 1095.979 & 0.288287806 & 0.125691222 \\
\hline O-Methyl-inositol & 1864 & 217.1000061 & 983.9855 & $0.00178648 *$ & 0.620371335 \\
\hline Lactic acid & 1068.7 & 190.0287144 & 375.828 & 0.415203838 & $0.001090602 *$ \\
\hline $\begin{array}{l}\text { Erythronic acid-1,4- } \\
\text { lactone }\end{array}$ & 1443.3 & 233.0652149 & 696.1045 & 0.326891652 & $0.007332597 *$ \\
\hline 2-oxoisocaproic acid & 1222 & 200.0661441 & 514.791 & 0.271244428 & $0.042610563 *$ \\
\hline Aconitic acid & 1759 & 229.0914541 & 917.6615 & 0.138857908 & 0.077081581 \\
\hline Beta-Alanine & 1436.1 & 174.0100886 & 686.804 & 0.444107553 & 0.390753624 \\
\hline $\begin{array}{l}\text { 3-Hydroxy-3- } \\
\text { methylglutaric acid }\end{array}$ & 1614.2 & 247.0881815 & 820.943 & 0.177902797 & $6.10981 \mathrm{E}-06^{*}$ \\
\hline Monomethylphosphate & 1186.9 & 241 & 484.196 & 0.092828664 & 0.145718477 \\
\hline Ethanolamine & 1275.2 & 174.094677 & 560.518 & 0.36326865 & 0.550823584 \\
\hline myo-inositol & 2126.8 & 305.1000061 & 1135.123 & 0.353687953 & 0.971863168 \\
\hline Guanosine & 2799 & 245.1000061 & 1454.6085 & 0.451105605 & 0.212666883 \\
\hline Guanine & 2144.8 & 352.1363513 & 1145.067 & 0.585056177 & 0.063581615 \\
\hline Octadecanoic acid & 2243.4 & 117 & 1196.418 & 0.507103826 & 0.730679107 \\
\hline
\end{tabular}


Table 2 Continued.

\begin{tabular}{|c|c|c|c|c|c|}
\hline Name & RI & $\mathrm{mz}$ & RT & $\begin{array}{l}\text { E4-48h vs E4-36h } \\
\text { pvalue }\end{array}$ & $\begin{array}{l}\text { E4-72h vs E4 36h } \\
\text { pvalue }\end{array}$ \\
\hline Uridine & 2477.4 & 217.1000061 & 1313.227 & 0.55274185 & 0.094154163 \\
\hline Insitol-1-phosphate & 2468.7 & 318.1174541 & 1307.646 & 0.564702188 & $0.00771979 *$ \\
\hline Pantothenic acid & 2014.2 & 103 & 1070.657 & $0.014817073^{*}$ & $0.014848554 *$ \\
\hline $\begin{array}{l}\text { 9-Octadecenoic acid (Z)-, } \\
\text { methyl ester }\end{array}$ & 2100.3 & 83 & 1121.075 & 0.435338099 & 0.179331639 \\
\hline Hexadecanoic acid & 2047.9 & 117 & 1091.156 & 0.65330602 & 0.166979561 \\
\hline Arabinitol & 1747.4 & 217.1000061 & 910.159 & 0.556402262 & 0.413829672 \\
\hline Galactinol & 2996.6 & 204.1000061 & 1539.9575 & 0.731129725 & 0.056946092 \\
\hline Ribonic acid & 1809.5 & 292.1000061 & 950.2715 & 0.216895205 & 0.28511511 \\
\hline Gentibiose & 2889.5 & 361.2000122 & 1489.088 & 0.745851062 & 0.86693461 \\
\hline $\begin{array}{l}\text { 9,12-(Z,Z)- } \\
\text { Octadecadienoic acid }\end{array}$ & 2213.3 & 81.00312156 & 1181.329 & 0.822735444 & $0.003821939 *$ \\
\hline Glucopyranose & 1668.9 & 204.0926929 & 862.3385 & 0.793181779 & $0.003520262 *$ \\
\hline 2-Ketogluconic acid & 1675 & 204.0926929 & 862.3385 & 0.793181779 & $0.003520262 *$ \\
\hline $\begin{array}{l}\text { 9,12-Octadecadienoic acid } \\
(\mathrm{Z}, \mathrm{Z})-\text {, methyl ester }\end{array}$ & 2094.8 & 81.00533547 & 1118.1115 & 0.807883392 & 0.687618647 \\
\hline Diisobutyl-phthalate & 1874.4 & 149 & 990.058 & 0.89381326 & $0.012687143^{*}$ \\
\hline Mannitol & 1967 & 217.1000061 & 1045.58 & 0.915989779 & 0.730533563 \\
\hline Nicotinamide & 1485.9 & 179 & 729.7865 & 0.914683716 & $0.000592659 *$ \\
\hline N-Acetylglutamic acid & 1539.8 & 84 & 767.3565 & 0.924296767 & 0.814638394 \\
\hline 9-(Z)-Octadecenoic acid & 2218.1 & 117 & 1183.579 & 0.952725947 & 0.056004474 \\
\hline Gluconic acid-1,5-lactone & 1915.6 & 220.1000061 & 1014.0675 & 0.957120253 & 0.061851091 \\
\hline Pyruvic acid & 1057.3 & 88.99606439 & 365.556 & 0.972667525 & 0.5084875 \\
\hline
\end{tabular}

Note: Differences showing P values less than 0.05 were considered statistically significant, ${ }^{*}$ These compounds were were considered statistically significan

In the current study, however, most fatty acids did not accumulate. In addition, no fatty acids were found by HCA (Fig. 3) suggesting no correlation with cleistothecium formation or maturation. Of course some critical trace fatty acid metabolites may not have been detected by GC-MS, so additional experiments are needed to investigate the functions of fatty acids in sexual development of E. cristatum.

The majority of correlations between amino acids and organic acids were positive (Fig. 4) as these metabolites were generally up regulated and subsequently then down regulated in parallel during development. In contrast, most correlations of amino acids and organic acids with alcohols and carbohydrates (mainly sugar metabolites) were negative, resulting in late up regulation of the latter groups. In addition, most correlations between organic acids and amino acids were negative, as were most between phosphate metabolites and both alcohols and sugar metabolites. Amine metabolites were positively correlated with most organic acids and amino acids but negatively correlated with most alcohols and sugar metabolites. From these correlations, it appears that organic acids and amino acids are critical regulators of early sexual development, while carbohydrates may be critical for cleistothecium formation and ripening (at $72 \mathrm{~h}$.). The functions of most fatty acids, however, remain obscure as none were identified by HCA and no significant metabolite-metabolite correlations were found. In addition to metabolite profile, metamorphosis of fungi is strongly affected by environmental factors including nutrient availability, temperature, osmolarity and light (Ge et al. 2016, Dyer \& O'Gorman 2012, Krijgsheld et al. 2013, Roze et al. 2010). As metabolites are the final downstream products of gene expression, it is likely that certain 
metabolites are critical mediators linking environmental changes to morphological adaptation. Transcriptomic and proteomic studies have shown that morphogenesis of some fungi is regulated by Ras, PKA, MAPK and HOG signaling pathways. Metabolites could activate these pathways, act as downstream effectors, or regulate expression of genes associated with these pathways (Brown et al. 1999, Murad et al. 2001, Maidan et al. 2005a, Pham et al. 2006, Han et al. 2011, Han et al. 2012).

Taken together, we present compelling evidence that morphogenesis of fungi is regulated by the metabolite profile. Indeed, these metabolites provide the essential materials for each stage in the sexual development of E. cristatum. High concentrations of organic acids, amino acids, and certain phosphate and amine metabolites promote ascogonium formation, while down regulation of these metabolites favors cleistothecium formation and ripening. Conversely, alcohol and carbohydrate accumulation favors cleistothecium formation while low levels favor formation of ascogonium.

\section{Acknowledgements}

National Natural Science Funds of China (31660021), grants from Guizhou Academy of Agriculture Science ([2016]028), grants from Guizhou Academy of Agriculture Science (GAASSP-2014/004), Guizhou province science and technology support program [2017]2561 and Department of Science and Technology of Guizhou Province ([2016]1056). We would like to acknowledge Bionovogene Co., Ltd., Suzhou, China for providing help in GC-MS measurements.

\section{References}

Ana TM, María SR, Jose FM, Lourdes C. 2016 - Nitric oxide synthesis by nitrate reductase is regulated during development in Aspergillus. Molecular Microbiology 99, 15-33.

Bino RJ, Hall RD, Fiehn O, Kopka J et al. 2004 - Potential of metabolomics as a functional genomics tool. Plant Science 9, 418-425.

Blomberg A, Adler L. 1989 - Roles of glycerol and glycerol-3-phosphate dehydrogenase (NAD+) in acquired osmotolerance of Saccharomyces cerevisiae. Journal of Bacteriology 171, 10871092.

Brown Jr DH, Giusani AD, Chen X, Kumamoto CA. 1999 - Filamentous growth of Candida albicans in response to physical environmental cues and its regulation by the unique CZF1 gene. Molecular Microbiology 34, 651-662.

Chen H, Fink GR. 2006 - Feedback control of morphogenesis in fungi by aromatic alcohols. Genes \& Development 20, 1150-1161.

Chen H, Fujita M, Feng QH, Clardy J et al. 2004 - Tyrosol is a quorum-sensing molecule in Candida albicans. Proceedings of the National Academy of Science of the United States of America 101, 5048-5052.

Christensen SA, Kolomiets MV. 2011 - The lipid language of plant-fungal interactions. Fungal Genetics and Biology 48, 4-14.

Dimitrios IT, Nancy PK. 2007 - Oxylipins as developmental and host-fungal communication signals. Trends in Microbiology 15, 109-118.

Ding MZ, Cheng JS, Xiao WH, Qiao B et al. 2009 - Comparative metabolomic analysis on industrial continuous and batch ethanol fermentation processes by GC-TOF-MS. Metabolomics 5, 229-238.

Ding MZ, Li BZ, Cheng JS, Yuan YJ. 2010 - Metabolome analysis of differential responses of diploidand haploid yeast to ethanol stress. OMICS: A Journal of Integrative Biology 5, 553561.

Dyer PS, O'Gorman CM. 2012 - Sexual development and cryptic sexuality in fungi: insights from Aspergillus species. Fems Microbiology Reviews 36, 165-192.

Etxebeste O, Garzia A, Espeso EA, Ugalde U. 2010 - Aspergillus nidulans asexual development: making the most of cellular modules. Trends in Microbiology 18, 569-576. 
Gancedo C, Gancedo JM, Sols A. 1968 - Glycerol metabolism in yeasts Pathways of utilization and production. European Journal of Biochemistry 5, 165-172.

Ge YY, Wang YC, Liu YX, Tan YM. 2016 - Comparative genomic and transcriptomic analyses of the Fuzhuan brick tea-fermentation fungus Aspergillus cristatus. BMC Genomics 17, 428441.

Han KH, Lee DB, Kim JH, Kim MS et al. 2003 - Environmental factors affecting development of Aspergillus nidulans. Journal of Microbiology 41, 34-40.

Han TL, Cannon RD, Villas-Boas SG. 2011 - The metabolic basis of Candida albicans morphogenesis and quorum sensing. Fungal Genetics and Biology 48, 747-763,

Han TL, Cannon RD, Villas-Bo^as SG. 2012 - Metabolome analysis during the morphological transition of Candida albicans. Metabolomics 8, 1204-1217.

Kinderlerer JL. 1993 - Fungal strategies for detoxification of medium chain fatty acids. International Biodeterioration and Biodegradation 32, 213-224.

Krijgsheld P, Bleichrodt R, van Veluw GJ, Wang F et al. 2013 - Development in Aspergillus. Studies in Mycology 74, 1-29.

Liu ZY, Qin J, Li NL. 1991 - Study of conditions of sporogenesis of Aspergillus chevalieri var intermedius in FuZhuan Tea. Southwest China Journal of Agricultural Sciences 4, 73-77.

Maidan MM, Larissa DR, Joke S, Simone E et al. 2005a - The G protein-coupled receptor Gpr1 and the Ga protein Gpa2 act through the cAMP-protein kinase A pathway to induce morphogenesis in Candida albicans. Molecular Biology of the Cell 16, 1971-1986.

Maidan MM, Thevelein JM, Van DP. 2005b - Carbon source induced yeast-to-hypha transition in Candida albicans is dependent on the presence of amino acids and on the G-protein-coupled receptor Gpr1. Biochemical Society Transactions 33, 291-293.

Mazur P, Nakanishi K, El-Zayat AAE, Champe SP. 1991 - Structure and synthesis of sporogenic psi factors from Aspergillus nidulans. Journal of the Chemical Society, Chemical Communications 20, 1486-1487.

Mo H, Xu X, Yan M, Zhu Y. 2005 - Microbiological analysis and antibacterial effects of the indigenous fermented Puer tea. Agro food industry hi-tech 16, 16-18.

Mo H, Zhu Y, Chen Z. 2008 - Microbial fermented tea-a potential source of natural food preservatives. Trends in food science and technology 19, 124-130.

Mohler RE, Tu BP, Dombek KM, Hoggard JC et al. 2008 - Identification and evaluation of cycling yeast metabolites in two-dimensional comprehensive gas chromatography time of flight mass spectrometry data. Joural of Chromatography. A 1186, 401-411.

Murad AMA, D'Enfert C, Gaillardin C, Tournu H et al. 2001 - Transcript profiling in Candida albicans reveals new cellular functions for the transcriptional repressors CaTup1, CaMig1 and CaNrg1. Molecular Microbiology 42, 981-993.

Nickerson KW, Atkin AL, Hornby JM. 2006 - Quorum sensing in dimorphic fungi: Farnesol and beyond. Applied and Environmental Microbiology 72, 3805-3813.

Pham TK, Chong PK, Gan CS, Wright PC. 2006 - Proteomic analysis of Saccharomyces cerevisiae under high gravity fermentation conditions. Journal of Proteome Research 5, 3411-3419.

Rao GD, Sui JK, Zhang JG. 2016 - Metabolomics reveals significant variations in metabolites and correlations regarding the maturation of walnuts, Biol Open 5, 829-836.

Roze LV, Anindya C, Maris L, Randolph MB et al. 2010 - Volatile profiling reveals intracellular metabolic changes in Aspergillus parasiticus: veA regulates branched chain amino acid and ethanol metabolism. BMC Biochemistry 11, 33-48.

Smith CA, Want EJ, O'maille G, Abagyan R et al. 2006 - XCMS: processing mass spectrometry data for metabolite profiling using nonlinear peak alignment, matching and identification. Analytical Chemistry 78, 779-787.

Wyatt TT, Leeuwen MRV, Wosten HAB, Dijksterhuis J. 2014 - Mannitol is essential for the development of stress resistant ascospores in Neosartorya fischeri (Aspergillus fischeri). Fungal Genetics and Biology 64, 11-24. 
Yu JH. 2010 - Regulation of development in Aspergillus nidulans and Aspergillus fumigatus. Mycobiology 38, 229-237.

Zhao RJ, Zheng YX 1995 - Study on the produce oils of mortierella of filamentous fungi. Acta Mycologica Sinica 14, 130-135. 